\title{
Towards Development of a Hybrid DSMC-CFD Method for Simulating Hypersonic Interacting Flows
}

\author{
Wen-Lan Wang*, Quanhua Sun ${ }^{\dagger}$ and Iain D. Boyd ${ }^{\ddagger}$ \\ Department of Aerospace Engineering \\ University of Michigan, Ann Arbor, MI 48109
}

\begin{abstract}
A preliminary hybrid particle-continuum computational framework for simulating hypersonic interacting flows is proposed. The framework consists of the direct simulation Monte Carlo-Information Preservation (DSMC-IP) method coupled with a NavierStokes solver. Since the DSMC-IP method provides the macroscopic information in each time step, determination of the continuum fluxes across the interface between the particle and continuum domains becomes straightforward. A hypersonic flow over a two-dimensional wedge is considered as an example and compared with pure particle calculations. The results show that this preliminary hybrid framework is promising but several issues are yet to be resolved.
\end{abstract}

\section{Introduction}

Hypersonic flows are of great interest in space vehicle design because the interactions of shock/shock and shock/boundary layer create high localized temperatures and associated extremely high heating rates in the interaction region. Experimental measurements under realistic conditions are difficult to conduct due to the technical difficulties and tremendous costs. Therefore, it is in general required to analyze hypersonic flows by means of computational methods.

Recently, for a set of hypersonic free stream conditions, biconic configurations and hollow cylinder/flares were extensively studied with different numerical approaches. ${ }^{1-5}$ In comparisons with experimental data, ${ }^{6}$ it was evident that the computational fluid dynamics (CFD) techniques based on the Navier-Stokes (NS) equations have better performance than the direct simulation Monte Carlo (DSMC) method ${ }^{7}$ in terms of the

\footnotetext{
*Graduate Student Research Assistant, AIAA Student Member (aerowwl@engin.umich.edu)

${ }^{\dagger}$ Graduate Student Research Assistant, AIAA Student Member (qsun@engin.umich.edu)

$¥$ Associate Professor, AIAA Senior Member (iainboyd@engin.umich.edu)

Copyright (C) 2002 by the American Institute of Aeronautics and Astronautics, Inc. All rights reserved.
}

capture of flow structures and the prediction of the separation region size. However, it was shown that the CFD methods constantly over predict the heat transfer on the fore body ahead of where separation occurs. Furthermore, it was demonstrated in Ref. 8 that adjacent to the body wall, especially near the cone tip, there is a strong thermal nonequilibrium effect, that is inconsistent with the basic assumptions of the NS equations. In addition, Roy et al. ${ }^{9}$ showed that DSMC and CFD do agree for conditions that are more rarefied than the experiments. Therefore, the objective of the present work is to develop a combined DSMC-CFD computational method for the physically accurate and numerically efficient analysis of this class of hypersonic flows.

Two primary issues must be taken into account in developing a combination of DSMC and CFD methods. We first need to know when to switch between the methods. Since it is well known that the NS equations are not valid under rarefaction conditions, it is general to use a continuum breakdown parameter as the criterion. For the hypersonic flows mentioned above, this issue has been investigated in our previous work ${ }^{10}$ and it is concluded that a proposed parameter

$$
\mathrm{Kn}_{\max } \equiv \max \left(\mathrm{Kn}_{D}, \mathrm{Kn}_{T}, \mathrm{Kn}_{V}\right)
$$

with a threshold value of 0.05 can best predict the regions where the Navier-Stokes equations fail. The Knudsen number in Eq. 1 is expressed as

$$
\mathrm{Kn}_{Q}=\frac{\lambda}{Q}|\nabla Q|,
$$

where $Q$ can be any flow property in general. Since continuum breakdown is often related to the transport phenomena of viscosity and heat transfer, we only consider the flow properties of density(D), temperature $(\mathrm{T})$ and magnitude of velocity $(\mathrm{V})$.

Another issue regarding the development of a hybrid method is the approach for information exchange at the interface between the DSMC and CFD domains. At the interface, macroscopic flow properties 
must be provided to the CFD method to evaluate the net fluxes and to the DSMC method to initialize the particles entering from the continuum domain into the rarefaction domain. Several approaches have been considered, such as the Marshak condition, ${ }^{11}$ the kinetic flux-vector splitting (KFVS) scheme ${ }^{12,13}$ and the adaptive mesh and algorithm refinement (AMAR) embedding a particle method. ${ }^{14}$ Unfortunately, a robust, multi-dimensional scheme that is capable of handling nonequilibrium, hypersonic compressed flows has not yet been accomplished.

The primary difficulty of the second issue results from the fact that the DSMC approach always inherits very strong statistical scatter unless the number of samples (either the simulation particles or number of sampling time steps, or a combination of the two) is large enough. Recently, the information preservation method (IP) was proposed to reduce the statistical scatter in low-speed, constant density flow systems. ${ }^{15}$ Since then, the method has been generalized to allow density and temperature variations and shown to be effective in solving microfluidic and micro-electromechanical system (MEMS) problems. ${ }^{16-18}$ One advantage of the IP method is that the macroscopic values of the flow field are known at any time, since the information in cells is updated for each time step. This eases the complexity of coupling the particle method with the continuum solver. A hybrid approach that combines the IP method and a NS solver has shown great progress in solving micro-scale gas flows. ${ }^{19}$ This motivates us in the present study to explore the IP method for hypersonic, interacting flows.

The layout of the paper is as follows. A brief description of the continuum approach will be presented first in the next section, followed by an introduction of the DSMC-IP method. A detailed explanation on how to combine these two methods together is provided in the section of Domain Coupling. A hypersonic flow over a two-dimensional wedge is considered to assess the new hybrid technique. In the last section, conclusions and suggestions for future work are provided.

\section{Numerical Schemes \\ Continuum Approach}

The Navier-Stokes equations in the continuum domain are solved numerically using an explicit GaussSeidel line relaxation method and second-order accurate, modified Steger-Warming flux vector splitting. ${ }^{20}$ The viscosity $\mu$ is modeled with the power law

$$
\mu=\mu_{\text {ref }}\left(T / T_{\text {ref }}\right)^{\omega}
$$

and the thermal conductivity $\kappa$ is determined from the Prandtl number

$$
\operatorname{Pr}=c_{p} \mu / \kappa
$$

where $c_{p}$ is the specific heat at constant pressure. A slip-boundary model proposed by Gökçen ${ }^{21}$ is incor- porated:

$$
\begin{aligned}
\vec{u}_{s} & =\left.\frac{2-\sigma_{v}}{\sigma_{v}} \lambda_{v} \frac{\partial \vec{u}}{\partial n}\right|_{w} \\
T_{s} & =T_{w}+\left.\frac{2-\sigma_{T}}{\sigma_{T}} \lambda_{T} \frac{\partial T}{\partial n}\right|_{w}
\end{aligned}
$$

where $T_{w}$ is the temperature of the surface, and $\sigma_{v}$ and $\sigma_{T}$ are the tangential momentum and thermal accommodation coefficients of the surface. Here the mean free path for momentum, $\lambda_{v}$, and thermal energy, $\lambda_{T}$ are defined as

$$
\lambda_{v}=\frac{2 \mu}{\rho \bar{c}}, \quad \lambda_{T}=\frac{2 \kappa}{\rho \bar{c} c_{v}}
$$

where $\bar{c}=\sqrt{8 R T / \pi}$ is the mean molecular speed, and $c_{v}$ is the specific heat at constant volume.

\section{DSMC-IP Approach}

The information preservation method was first developed by Fan and Shen ${ }^{15}$ to overcome the statistical scatter problem in DSMC simulations, especially for systems in which the flow speed is much smaller than the molecular speed. In addition to the ordinary thermal velocity that is utilized to compute the particle trajectory, each simulation particle in the DSMC-IP method also possesses macroscopic preserved information such as velocity vector and temperature. The DSMC-IP method has achieved great success for solving micro-scale gas flows (see Refs. 16-18).

In the most recent work by Sun and Boyd, ${ }^{22}$ an additional temperature term is introduced in order to solve the contradiction between the real flux and the DSMC-IP representation of the translational energy flux across a cell interface. From gas kinetic theory, the average translational energy of a molecule at equilibrium temperature $T$ is $3 k T / 2$ ( $k$ is the Boltzmann constant), whereas the average translational energy carried by a molecule across an interface is $2 k T$. The extra energy must be correctly modeled or the energy of the whole system will not be conserved. Therefore, the simulation particles in the IP method have an additional temperature $T_{a}$.

In each time step of the DSMC-IP method, simulation particles are first moved and collided in the usual way as in the standard DSMC method. The preserved velocity in the $r_{i}$ direction and temperature of simulation particles are updated by solving

$$
\begin{aligned}
& \frac{\partial V_{i}}{\partial t}=-\frac{1}{\rho_{c}} \frac{\partial p_{c}}{\partial r_{i}} \\
& \frac{\partial}{\partial t}\left(\frac{V_{i}^{2}}{2}+\frac{\xi \cdot R \cdot T}{2}\right)=-\frac{1}{\rho_{c}} \frac{\partial}{\partial r_{i}}\left(V_{i, c} \cdot p_{c}\right)
\end{aligned}
$$

where $p$ is the pressure, $\rho$ is the mass density, $\xi$ is the number of internal degrees of freedom of molecules, and the subscript $c$ denotes the macroscopic information for the computational cells. After the preserved 
information of simulation particles is updated, the preserved information for cells is updated by taking the arithmetic average over the information of all $N_{p}$ particles in the cell.

$$
\begin{aligned}
& V_{i, c}=\frac{1}{N_{p}} \sum_{j=1}^{N_{p}} V_{i, j} \\
& T_{c}=\frac{1}{N_{p}} \sum_{j=1}^{N_{p}}\left(T_{j}+T_{a, j}\right)
\end{aligned}
$$

The density is updated by solving the continuity equation

$$
\frac{\partial \rho_{c}}{\partial t}=-\frac{\partial}{\partial r_{i}}\left(\rho_{c} \cdot V_{i, c}\right) .
$$

The ideal gas law, $p=\rho R T$, is assumed. A detailed description and implementation of the DSMC-IP method can be found in Ref. 22 .

Note that an adequate numerical scheme must be employed to solve Eq. 8 due to the presence of shock waves in supersonic flows. Since the same equation also appears in the NS equations, we use the same technique described in the last subsection for solving it.

The current DSMC-IP code is based on a parallel optimized DSMC code named MONACO. ${ }^{23}$ A sub-cell scheme is implemented for selection of collision pairs where the number of sub-cells is scaled by the local mean free path.

\section{Domain Coupling}

To implement the coupling between the particle method and the NS solver, reservoir DSMC-IP cells are introduced in the continuum domain adjacent to the domain interface, as depicted in Fig. 1. A similar concept of reservoir cells was first proposed in Ref. 24.

The reservoir DSMC-IP cells work as an extension of the particle domain. There are two levels of reservoir cells. Simulation particles that end their movement phase within the pure particle domain or the L1 reservoir level are retained. Those that leave these two regions are removed. For each time step, all simulation particles in the L2 reservoir cells are first deleted and then re-generated based on the cell-centered values. The number of new particles is evaluated from the cell density value and the particle velocities and temperature are initialized to the Chapman-Enskog distribution $^{25}$ based on the corresponding cell values. The newly generated particles are randomly distributed within the L2 cells. In this study, one layer of L1 cells and five layers of L2 cells are employed.

In the continuum domain, the NS solver determines the interface continuum fluxes by using the NS variables and DSMC-IP cell macroscopic information. Since the macroscopic information in the DSMC-IP cells is known in each time step, the DSMC-IP cells adjacent to the domain interface are treated as the
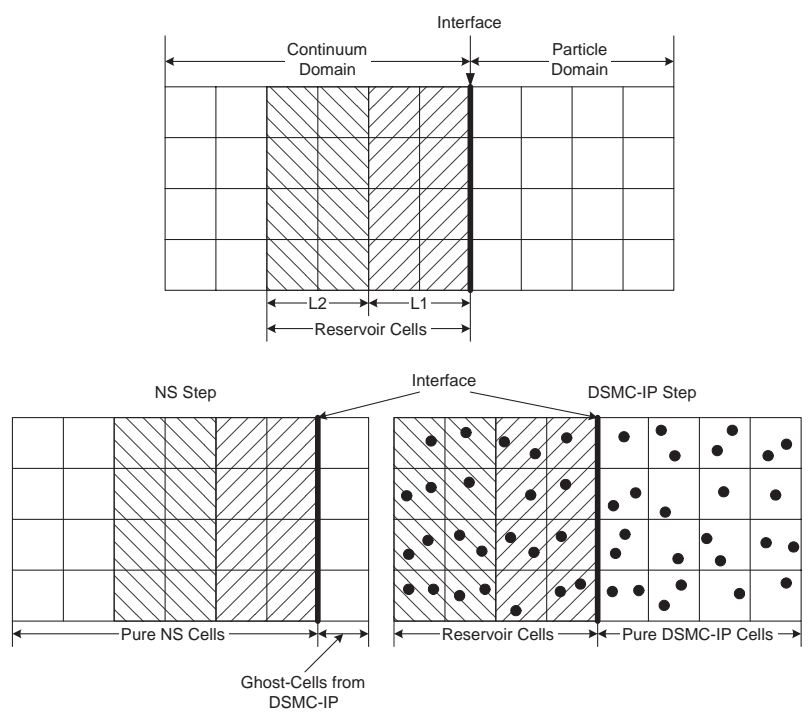

Fig. 1 Interface cell types.

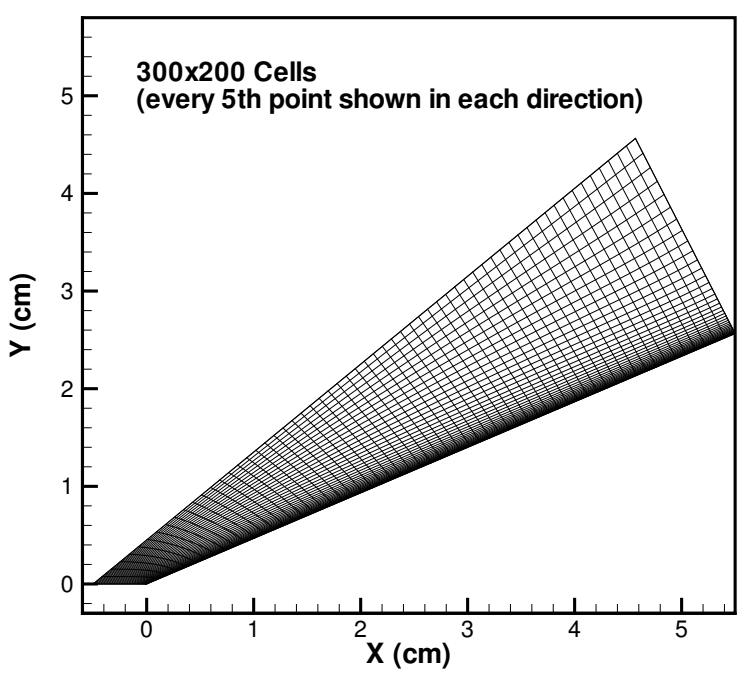

Fig. 2 Grid employed for $25^{\circ}$ wedge.

ghost cells that provide the boundary conditions for flux computations.

\section{Numerical Example}

This section describes the numerical experiment that is performed to assess the hybrid technique. A geometry of a $2 \mathrm{D}$ wedge with a $25^{\circ}$ half angle is chosen. The symmetric line of the wedge is aligned with the free-stream. Therefore, only the upper half of the wedge is considered. Since the wake region behind the wedge is not of interest in this investigation, we assume that the wedge is infinitely long but only the first $5 \mathrm{~cm}$ from the leading edge is considered. A structured grid, 300 cells along the body by 200 cells normal to the body, used in all our computations is shown in Fig. 2.

The fluid is pure nitrogen and the free-stream conditions are listed in Table 1. The mean-free-path in the free-stream is about $10^{-4} \mathrm{~m}$, accordingly. These 
specific free-stream conditions are the same as those in CUBRC Run $28^{26}$ except for a lower flow speed.

In the CFD calculation, $\mu_{\text {ref }}=1.656 \times 10^{5} \mathrm{~N} \cdot \mathrm{s} / \mathrm{m}^{2}$, $T_{\text {ref }}=273 \mathrm{~K}$ and $\omega=0.74$. The Prandtl number is considered as a constant of 0.72 . An isothermal wall at a temperature of $293.3 \mathrm{~K}$ is assumed.

Table 1 Free-stream conditions of numerical experiment.

\begin{tabular}{cccc}
\hline \hline$U_{\infty}(\mathrm{m} / \mathrm{s})$ & $T_{\infty}(\mathrm{K})$ & $\rho_{\infty}\left(\mathrm{kg} / \mathrm{m}^{3}\right)$ & $M_{\infty}$ \\
\hline 1111.1 & 185.6 & $6.545 \times 10^{-4}$ & 4.00 \\
\hline
\end{tabular}

A pure DSMC steady state solution is obtained with the use of more than 1.8 million simulation particles at the end of the computation. The reference time step in the pure DSMC calculation is 5 nsec. 300,000 time steps of computation are performed and the last 50,000 time steps are sampled to obtain the results.

In Fig. 3(a), it is shown that the number of particles per cell is generally greater than 10 . The ratio of the characteristic cell size $\Delta s$ to the local mean free path $\lambda$ is displayed in Fig. 3(b). The characteristic cell size is defined as the cell length scale in the direction of maximum gradient of density, pressure, temperature and velocity. The DSMC method requires this ratio be less than unity. It is clear that this criterion is not satisfied in most regions except in the region very close to the wedge surface. However, since a sub-cell scheme is implemented in the DSMC method and there are a large number of particles per cell in most regions, adequate spatial resolution should be provided.

In this hybrid simulation, a steady state solution from the CFD method is first obtained. Using the steady state solution and $\mathrm{Kn}_{\max }$, one can determine the locations of the interfaces between the continuum and the particle domains. In the particle region, cell values are set to the CFD steady state results and simulation particles are initialized to the ChapmanEnskog distribution.

Although it has been concluded in the previous work $^{10}$ that $\mathrm{Kn}_{\max }=0.05$ is a good criterion for separating the two domains, a more conservative criterion of $\mathrm{Kn}_{\max }=0.02$ is actually employed in this investigation. The initial particle domain and the domain after 400,000 hybrid simulation iterations are illustrated in the shaded areas of Figs. 4. About $40.2 \%$ of the total number of cells are in the particle domain at the beginning. This value increases gradually with time and reaches about $44.8 \%$ in the end. Each cell in the particle domain has about 70 simulation particles on average. The reference time step in the hybrid computation is 1 nsec. The hybrid method results presented in this paper are obtained by sampling the last 20,000 time steps.

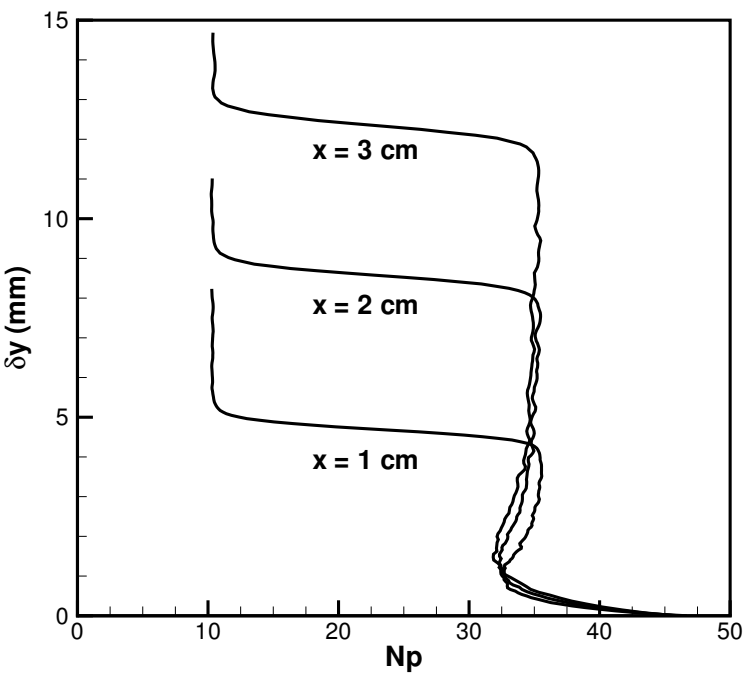

a) Number of particles per cell along three different constant- $x$ coordinates.

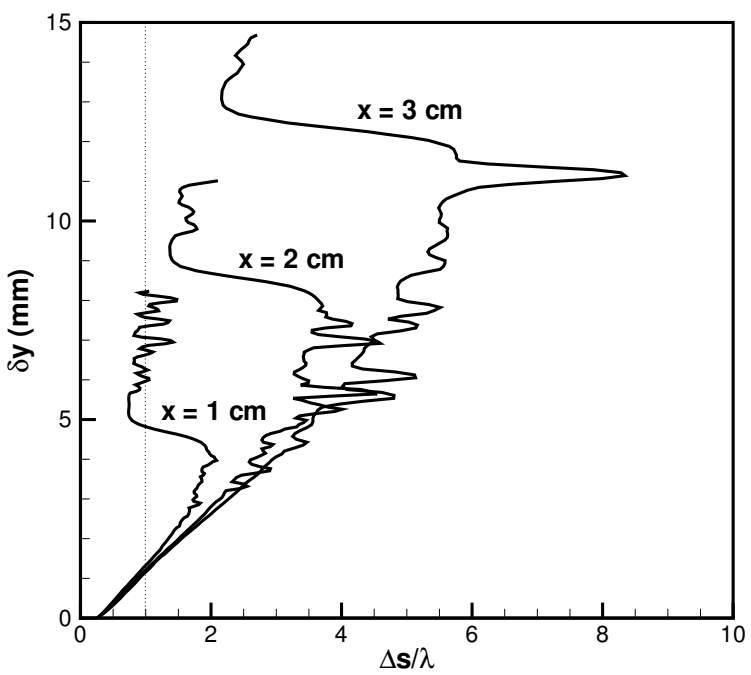

b) Ratio of simulation cell size in direction of maximum gradient to local mean free path along three different constant- $x$ coordinates.

Fig. 3 Variation of the numerical parameters along three vertical paths.

\section{Flow Fields}

Comparisons of the density and translational temperature contours obtained with pure DSMC and the hybrid methods are made in Figs. 5(a) and 5(b), respectively. The hybrid method results are in good agreement with the pure DSMC results, in general. Additionally, the hybrid method provides much smoother results.

\section{Surface Properties}

In Figs. 6, comparisons of flow properties along the wedge surface are made. Generally speaking, the flow properties predicted by the hybrid method along the wall are in excellent agreement with the DSMC solu- 


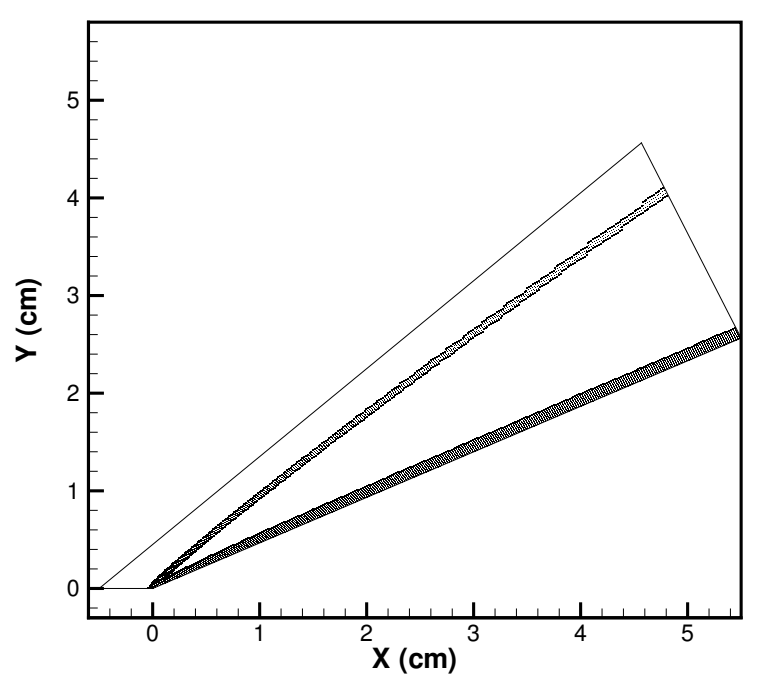

a) Initial particle domain.

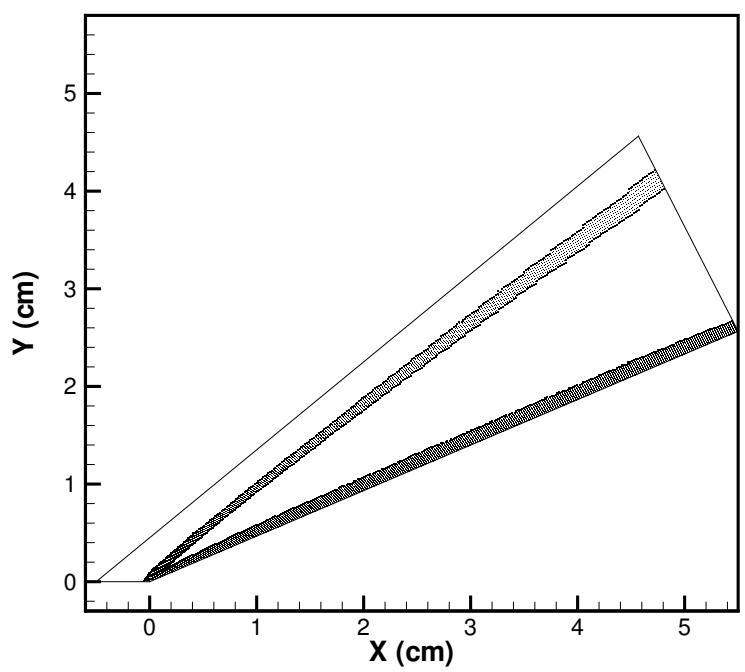

b) Particle domain after 400,000 iterations.

Fig. 4 Particle domain in hybrid simulation.

tions. The Stanton number in Fig. 6(a) is defined as

$$
S t=\frac{q_{w}}{\frac{1}{2} \rho_{\infty} U_{\infty}^{3}} .
$$

It is interesting to note that the heat transfer rate predicted by the hybrid method slightly shifts away from the initial CFD result that is almost identical to the DSMC result. A similar behavior is also observed for the skin friction coefficient in Fig. 6(b). In contrast to heat transfer rate and skin friction, pressure along the wedge surface computed with the hybrid method moves towards the DSMC result, as shown in Fig. 6(c).

\section{Detailed Comparisons}

In Fig. 7, the pure DSMC, CFD and hybrid solutions are compared in detail along a line normal to the wedge at $x=0.5 \mathrm{~mm}$. Since this location is very close to the wedge leading edge, where the flow is strongly nonequilibrium, the CFD and pure DSMC solutions

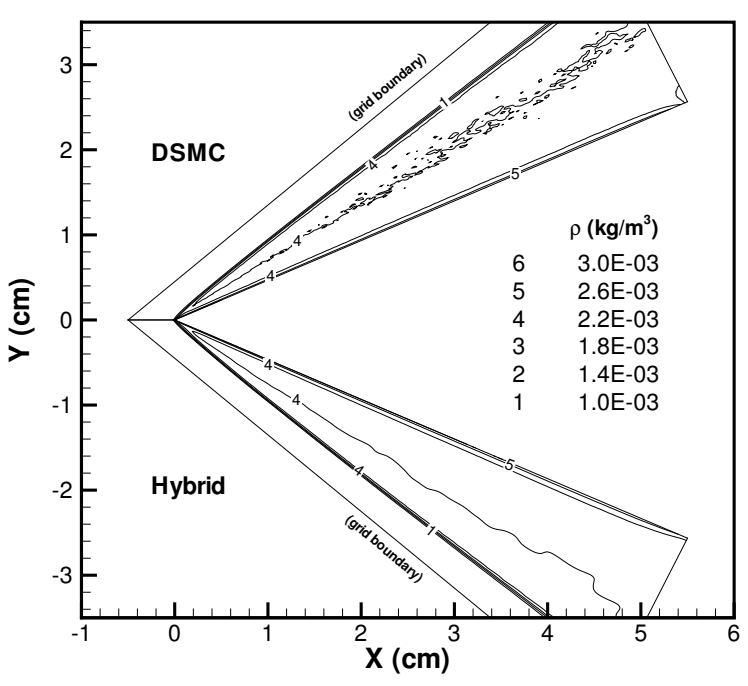

a) Comparison of mass density.

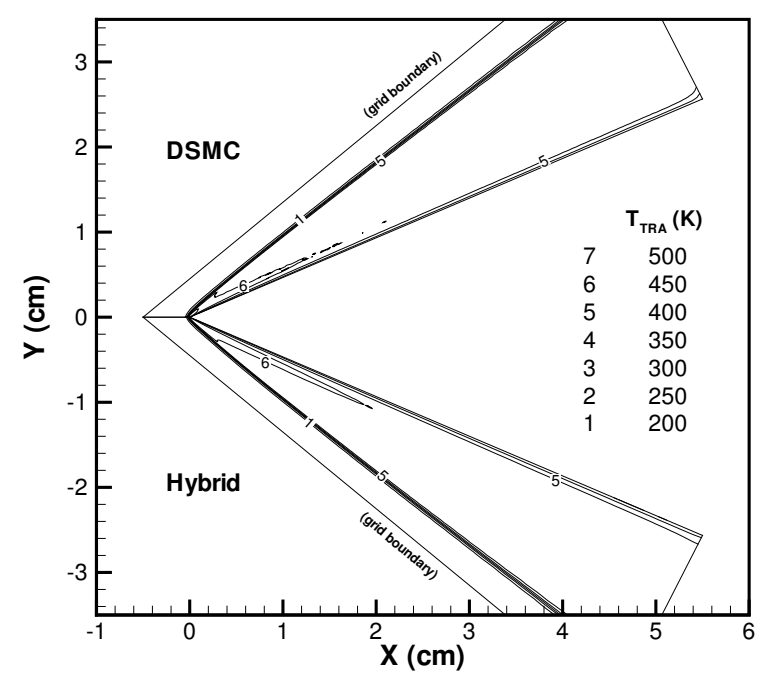

b) Comparison of translational temperature.

Fig. 5 Comparison of DSMC and hybrid solutions of mass density and translational temperature.

are therefore different to a large extent. It is evident that in the region very close to the wall $(\delta n<0.15$ $\mathrm{mm}$ ), the density and velocity obtained with the hybrid approach are in outstanding agreement with the pure DSMC results. Overall, they both move from the initial CFD conditions towards the DSMC solutions and are qualitatively consistent with the DSMC solutions. However, the translational temperature predicted by the hybrid approach is very different to the DSMC results. The reason for this poor result may come from the translational energy model and will be discussed later. The horizontal dotted line in Fig. 7 indicates the interface between the particle region (lower portion) and continuum region (upper portion).

A similar comparison between the pure DSMC, CFD and hybrid solutions for density, velocity and translational temperature at $x=1.0 \mathrm{~mm}$ is displayed in Fig. 8. It is clear that the hybrid technique works 


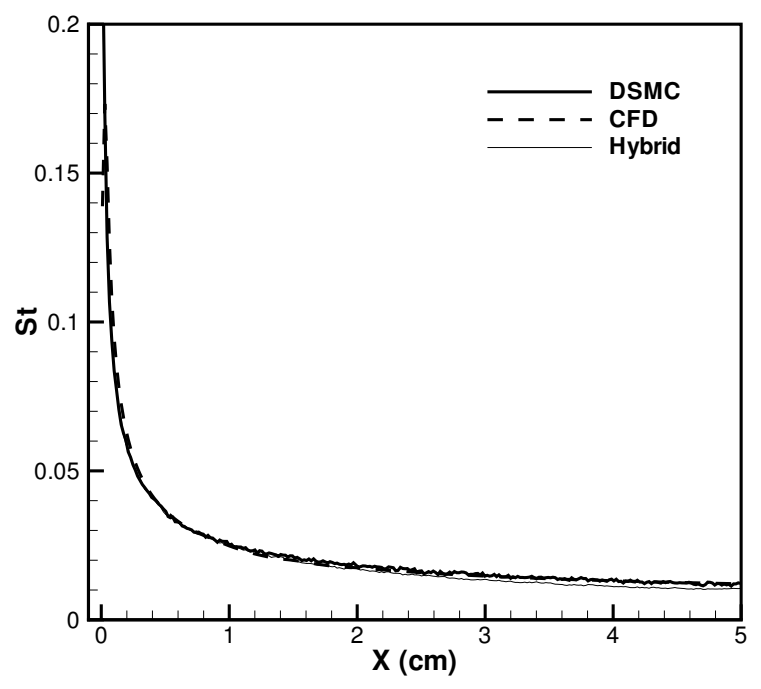

a) Comparison of surface heat transfer rate.

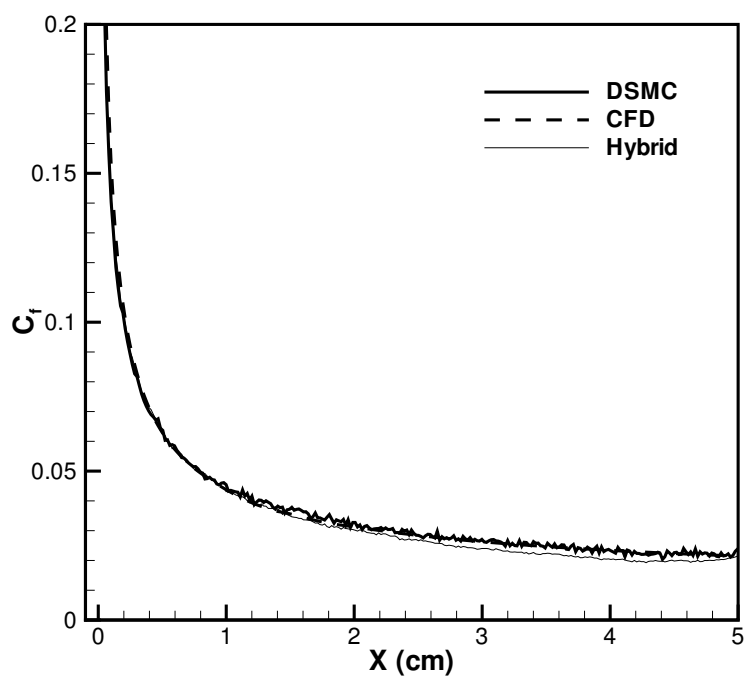

b) Comparison of skin friction coefficient.

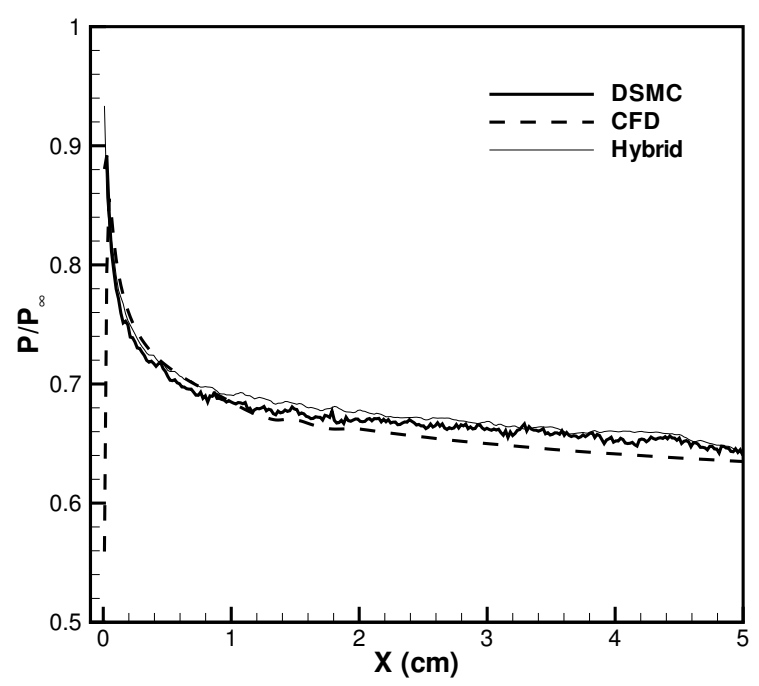

c) Comparison of surface pressure.

Fig. 6 Comparison of surface properties with different numerical approaches.

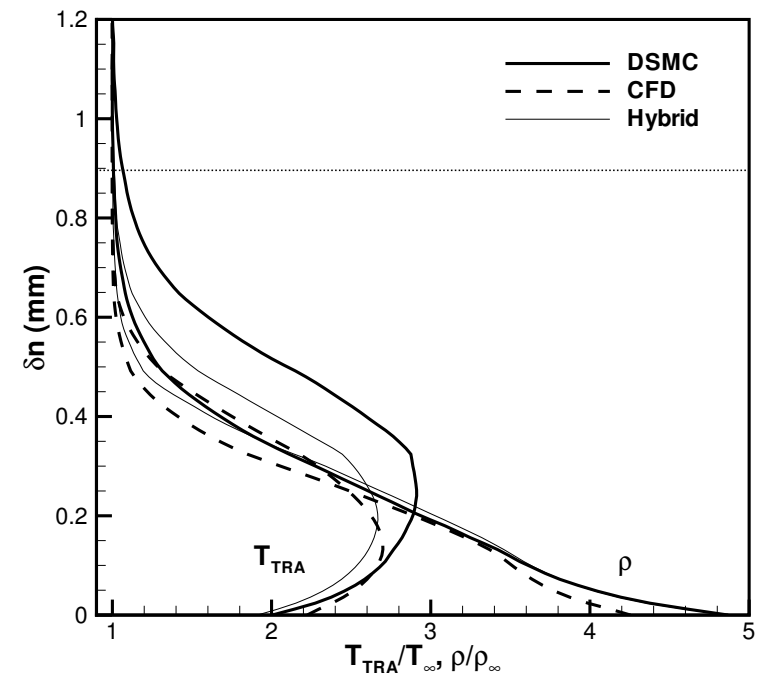

a) Comparison of temperature and density.

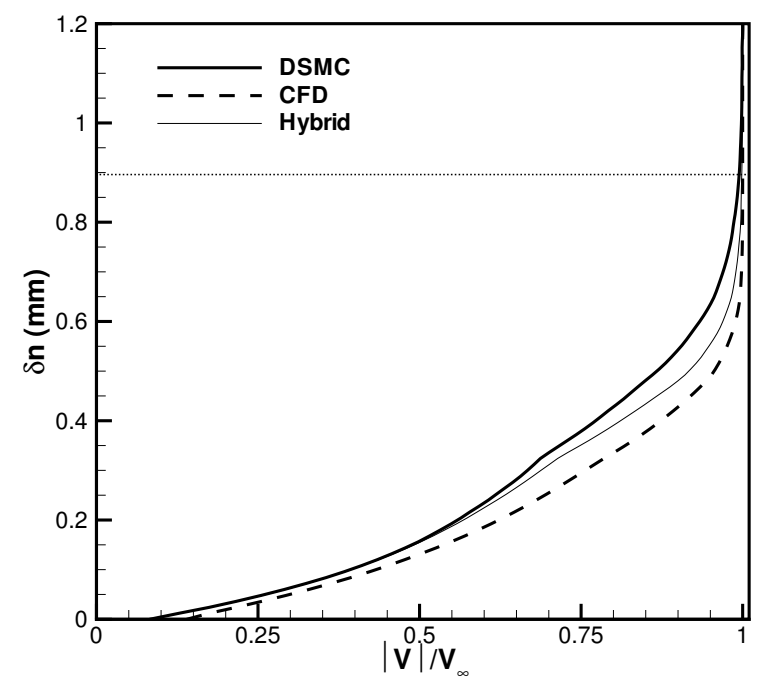

b) Comparison of velocity.

Fig. 7 Profiles along the line normal to the wedge surface at $\mathrm{x}=0.5 \mathrm{~mm}$.

fairly well in computing the density and velocity but poorly in modeling the translational temperature.

Further downstream at $x=5.0 \mathrm{~mm}$ where the nonequilibrium effect starts to weaken, the NavierStokes equations coupled with a slip-boundary model perform very well. As shown in Fig. 9, the CFD and DSMC solutions approach one another, especially in the region adjacent to the wall.

The whole computational domain at this location is divided into four regions along the $\delta n$ direction: particle (I: $0<\delta n<0.8$ ), continuum (II: $0.8<\delta n<1.86$ ), particle (III: $1.86<\delta n<2.6$ ) and continuum (IV: $\delta n>2.6)$. It is shown in Fig. 9(a) that the density and translational temperature predicted by the hybrid approach are in fair agreement with the pure DSMC solutions, except near the interface between regions II and III. The velocity profile in Fig. 9(b) exhibits unexpected behavior at the interface between regions I 


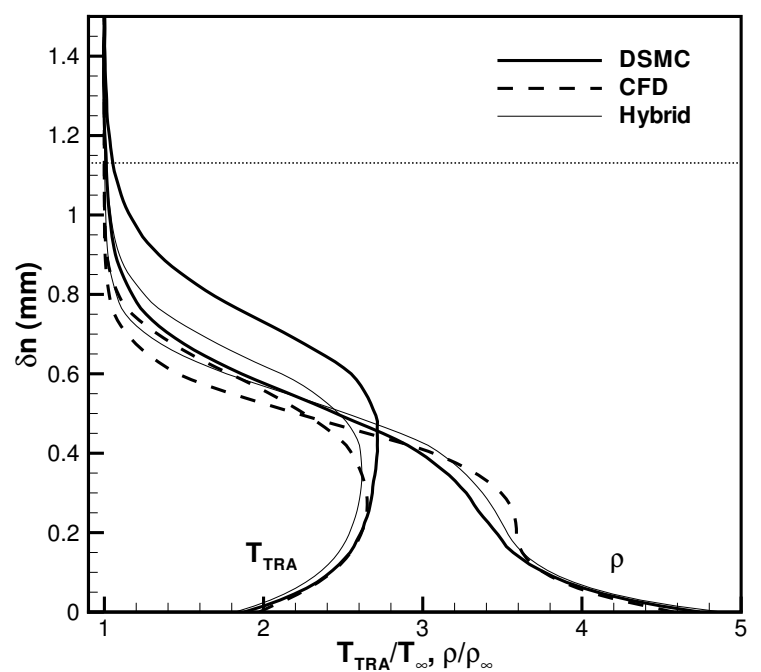

a) Comparison of temperature and density.

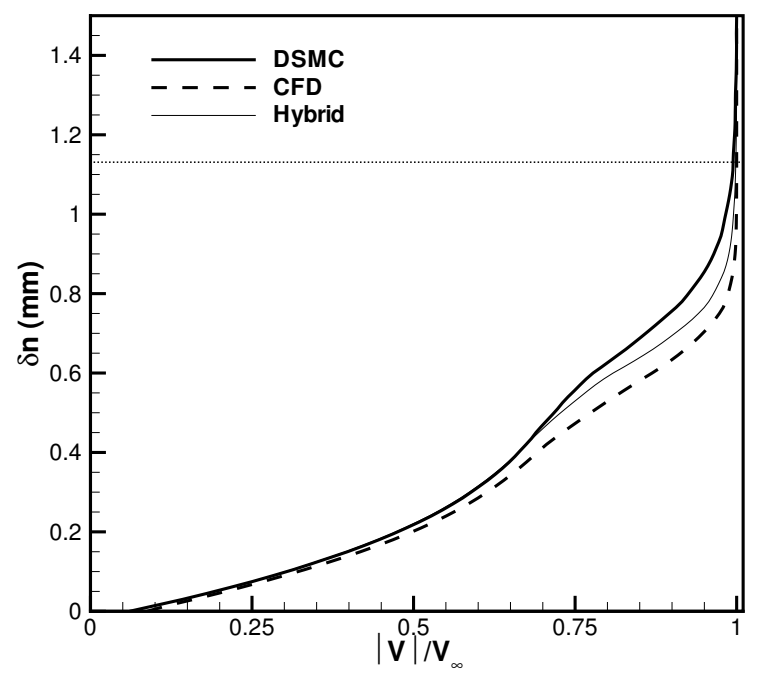

b) Comparison of velocity.

Fig. 8 Profiles along the line normal to the wedge surface at $x=1.0 \mathrm{~mm}$.

and II. The reasons for that may be due to the size of region III being too small or the numerical scheme employed to solve Eq. 8 in the particle regions, and will be discussed later.

Comparisons of density, translational temperature and velocity profiles obtained with different methods along the line normal to the wedge at $x=1.0 \mathrm{~cm}$ and $2.0 \mathrm{~cm}$ are plotted in Figs. 10 and 11, respectively. In general, the results calculated by using the hybrid approach qualitatively agree with the pure DSMC results and have the same problems as in Fig. 9.

\section{Discussion}

There are three main issues regarding the hybrid method results. First, it is clear that the breakdown criterion $\mathrm{Kn}_{\max }=0.02$ does not capture the shock front very accurately, causing the particle region near the shock to be too small and in turn causing the hy-

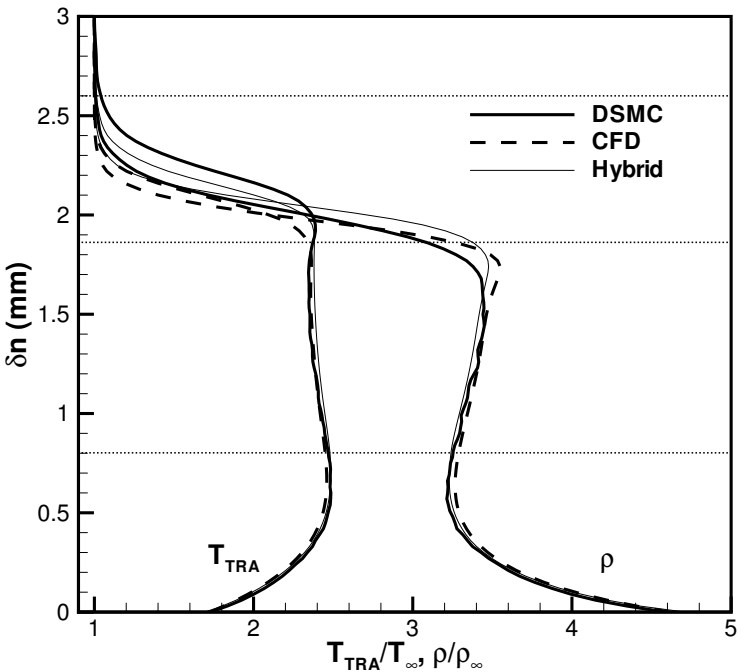

a) Comparison of temperature and density.

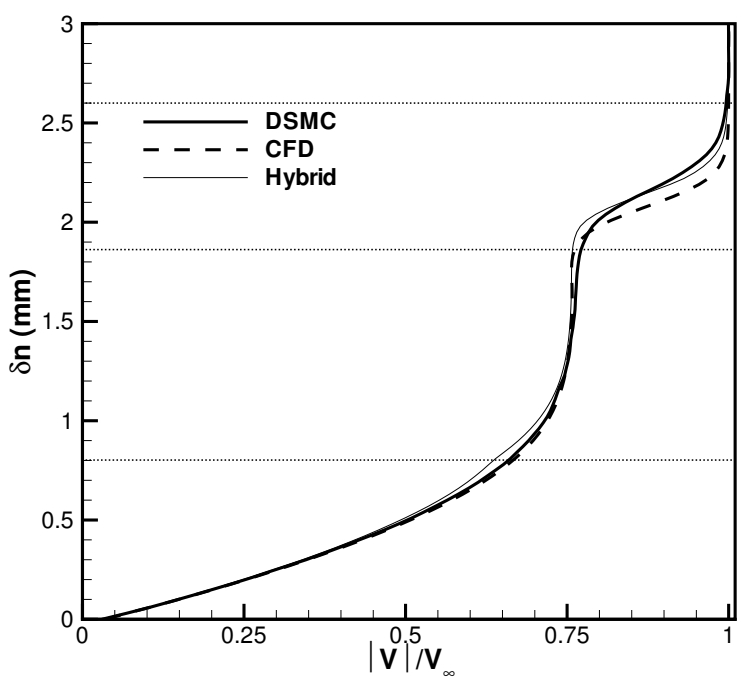

b) Comparison of velocity.

Fig. 9 Profiles along the line normal to the wedge surface at $x=0.5 \mathrm{~cm}$.

brid technique to have difficulties to accurately predict the shock structures. As a result, it is difficult for the continuum method after the shock to obtain the correct solution because the DSMC-IP method provides a questionable boundary condition.

The second issue occurs in the region very close to the wedge leading edge where the translational temperature is not correctly predicted by the DSMC-IP method. As mentioned in the Numerical Schemes section, the DSMC-IP method has an additional temperature term for balancing the energy transported by a molecule across an interface. However, this extra energy estimated in the current DSMC-IP method is based on the equilibrium assumption in which the velocity distribution function is Maxwellian. Because of the strong nonequilibrium near the leading edge, the extra energy modeled in the DSMC-IP technique is no longer valid. Consequently, the translational tempera- 


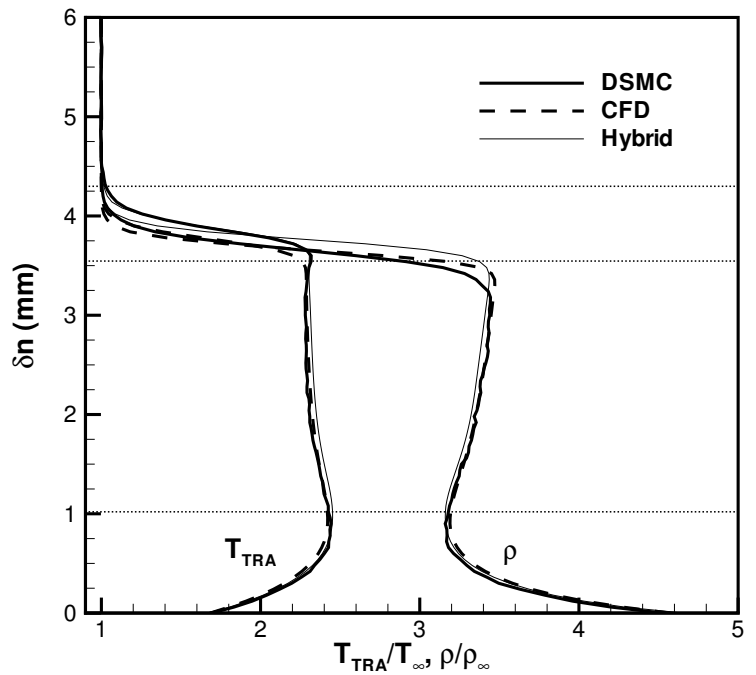

a) Comparison of temperature and density.

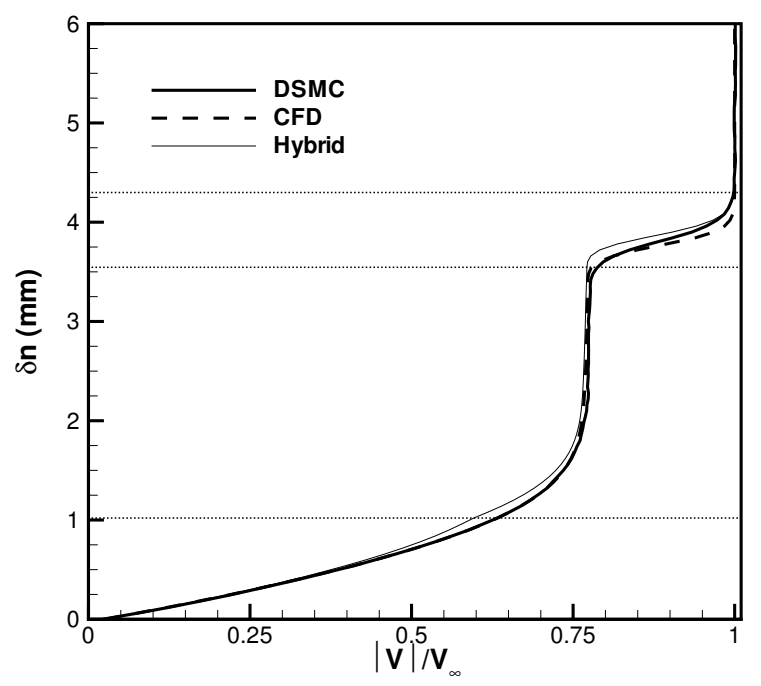

b) Comparison of velocity.

Fig. 10 Profiles along the line normal to the wedge surface at $x=1.0 \mathrm{~cm}$.

ture is poorly predicted by the DSMC-IP approach in the particle region.

The hybrid velocity profiles in Figs. 9 to 11 is the third concern. The reason for the unexpected behavior may be attributed to the first issue, or it may be a result of other factors, such as the way we solve the continuity equation Eq. 8 in the particle region. In this study, we adopt the same numerical scheme as used in the CFD code to solve Eq. 8. On the cell interfaces, the way that the specific numerical scheme in CFD calculates the mass flux largely depends on the characteristic equations which are obtained from the Euler equations. By definition, neither the Euler nor the Navier-Stokes equations are valid in the continuumbreakdown region. As a result, using any techniques derived from them may induce errors.

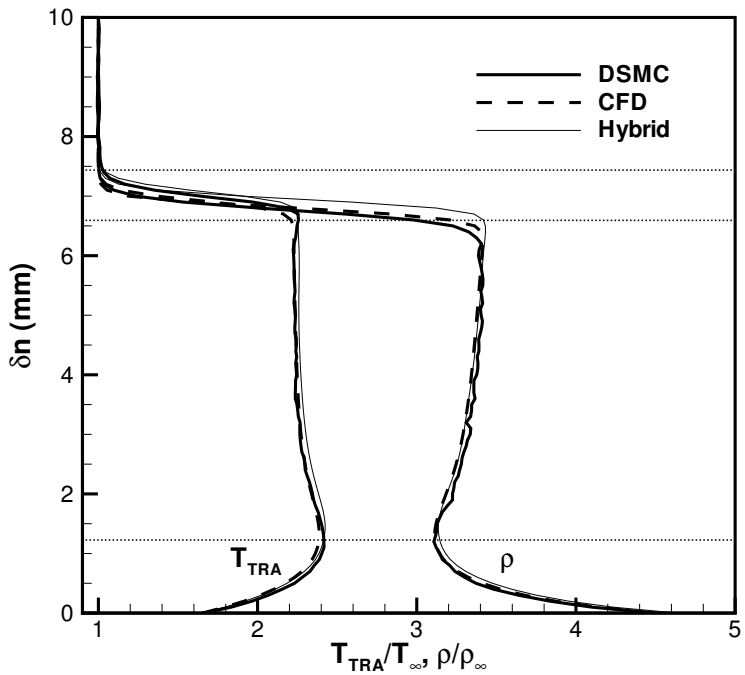

a) Comparison of temperature and density.

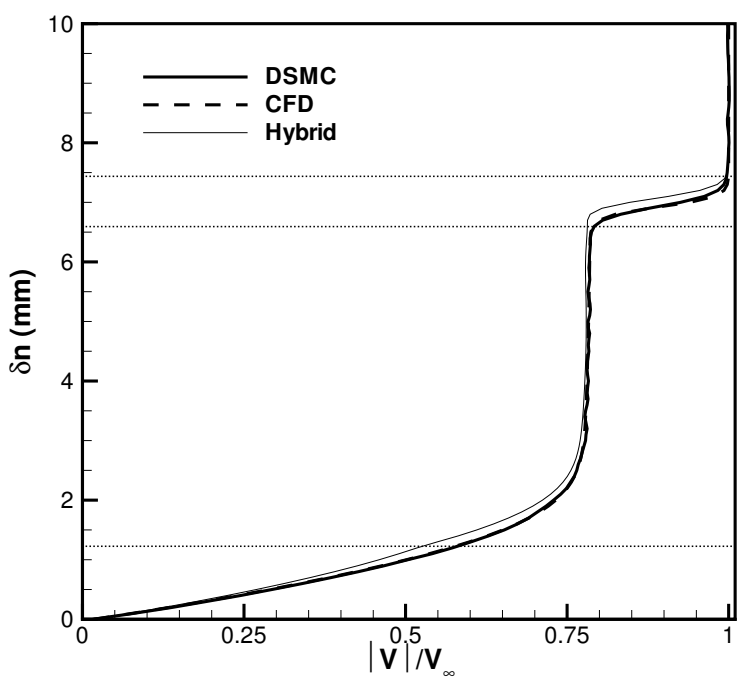

b) Comparison of velocity.

Fig. 11 Profiles along the line normal to the wedge surface at $x=2.0 \mathrm{~cm}$.

\section{Concluding Remarks and Future Work}

We have described a preliminary hybrid particlecontinuum method for computing hypersonic, interacting flows. In the particle domain, the DSMC-IP technique was employed while in the continuum domain, a finite volume, second-order accurate, StegerWarming flux vector splitting NS solver was utilized. Since the macroscopic values of the flow field in the DSMC-IP approach are updated in cells for each time step advanced, coupling these two domain becomes straightforward. Information exchange on the interface between the particle and continuum domains was continuously carried out for each time step.

A numerical experiment of a hypersonic flow over a wedge was conducted, with the use of the continuumbreakdown criterion $\mathrm{Kn}_{\max }=0.02$. The hybrid method was first initialized with a steady state CFD solution and then marched forward. It is concluded 
that the new hybrid method is able to simulate the hypersonic flow very well, generally speaking. The detailed comparisons showed that the density and velocity computed with the hybrid method matched with the exact (pure DSMC) solutions over the entire flow field faithfully, but translational temperature was poorly modeled near the leading edge.

The poor performance of the hybrid method on predicting the translational temperature around the tip of the wedge is primarily caused by the equilibrium approximation in the DSMC-IP technique to estimate the extra translational energy carried by a molecule moving from one cell to the other. A variant of this equilibrium model is under investigation.

An adequate numerical scheme for solving the continuity equation in the particle region is necessary as the Euler and Navier-Stokes equations fail in the continuum-breakdown region and consequently any numerical schemes derived from these equations may fail in this region.

The numerical experiment also showed that the size of the particle region around the shock wave is too small using the current breakdown criterion. Some portion of the shock front was not included in the particle domain. This aspect may lead to the unexpected behavior observed in the velocity profile. A more conservative criterion would help to solve this problem but would also reduce the numerical efficiency.

\section{Acknowledgments}

The authors gratefully acknowledge Professor Graham Candler of University of Minnesota for providing the CFD code. The views and conclusions contained herein are those of the authors and should not be interpreted as necessarily representing the official policies or endorsements, either expressed or implied, of the AFOSR or the U.S. Government. The authors would also like to express thanks for the assistance of the Minnesota Supercomputing Institute.

This work was sponsored by the Air Force Office of Scientific Research under grant F49620-01-1-0003.

\section{References}

${ }^{1}$ Candler, G. V., Nompelis, I., and Druguet, M.-C., "NavierStokes Predictions of Hypersonic Double-Cone and CylinderFlare Flow Field," AIAA Paper 2001-1024, Jan. 2001.

${ }^{2}$ Gnoffo, P. A., "CFD Validation Studies for Hypersonic Flow Prediction," AIAA Paper 2001-1025, Jan. 2001.

${ }^{3}$ Moss, J. N., "DSMC Computations for Regions of Shock/Shock and Shock/Boundary Layer Interaction," AIAA Paper 2001-1027, Jan. 2001.

${ }^{4}$ Boyd, I. D. and Wang, W.-L., "Monte Carlo Computations of Hypersonic Interacting Flows," AIAA Paper 2001-1029, Jan. 2001.

${ }^{5}$ Roy, C. J., Bartel, T. J., Gallis, M. A., and Payne, J. L., "DSMC and Navier-Stokes Predictions for Hypersonic Laminar Interacting Flows," AIAA Paper 2001-1030, Jan. 2001.

${ }^{6}$ Harvey, J. K., Holden, M. S., and Wadhams, T. P., "Code Validation Study of Laminar Shock/Boundary Layer and
Shock/Shock Interactions in Hypersonic Flow. Part B: Comparison with Navier-Stokes and DSMC Solutions," AIAA Paper 2001-1031, Jan. 2001.

${ }^{7}$ Bird, G. A., Molecular Gas Dynamics and the Direct Simulation of Gas Flows, Oxford University Press, Oxford, Oxford, 1994.

${ }^{8}$ Wang, W.-L., Boyd, I. D., Candler, G. V., and Nompelis, I., "Particle and Continuum Computations of Hypersonic Flow Over Sharp and Blunted Cones," AIAA Paper 2001-2900, June 2001.

${ }^{9}$ Roy, C. J., Gallis, M. A., Bartel, T. J., and Payne, J. L., "Navier-Stokes and DSMC Simulations for Hypersonic Laminar Shock-Shock Interaction Flows," AIAA Paper 2002-0737, Jan. 2002.

${ }^{10}$ Wang, W.-L. and Boyd, I. D., "Continuum Breakdown in Hypersonic Viscous Flows," AIAA Paper 2002-0651, Jan. 2002.

${ }^{11}$ Hash, D. B. and Hassan, H. A., "Assessment of Schemes for Coupling Monte Carlo and Navier-Stokes Solution Methods," Journal of Thermophysics and Heat Transfer, Vol. 10, No. 2, 1996, pp. 242-249.

${ }^{12}$ Chou, S. Y. and Baganoff, D., "Kinetic Flux-Vector Splitting for the Navier-Stokes Equations," Journal of Computational Physics, Vol. 130, No. 2, 1997, pp. 217-230.

${ }^{13}$ Lou, T., Dahlby, D. C., and Baganoff, D., "A Numberical Study Comparing Kinetic Flux-Vector Splitting for the Navier-Stokes Equations with a Particle Method," Journal of Computational Physics, Vol. 145, No. 2, 1998, pp. 489-510.

${ }^{14}$ Garcia, A. L., Bell, J. B., Crutchfield, W. Y., and Alder, B. J., "Adaptive Mesh and Algorithm Refinement Using Direct Simulation Monte Carlo," Journal of Computational Physics, Vol. 154, No. 1, 1999, pp. 134-155.

${ }^{15}$ Fan, J. and Shen, C., "Statistical Simulation of LowSpeed Unidirectional Flow in Transition Regime," Proceedings of the 21th International Symposium on Rarefied Gas Dynamics, Marseille, France, edited by e. a. R. Brum, 1998, p. 245.

${ }^{16} \mathrm{Cai}$, C., Boyd, I. D., Fan, J., and Candler, G. V., "Direct Simulation Methods for Low-Speed Microchannel Flows," Journal of Thermophysics and Heat Transfer, Vol. 14, No. 3, 2000, pp. $368-378$.

${ }^{17}$ Fan, J., Boyd, I. D., Cai, C.-P., Hennighausen, K., and Candler, G. V., "Computation of Rarefied Gas Flows Around a NACA 0012 Airfoil," AIAA Journal, Vol. 39, 2001, pp. 618-625.

${ }^{18}$ Sun, Q., Boyd, I. D., and Candler, G. V., "Numerical Simulation of Gas Flow OVer Micro-Scale Airfoils," AIAA Paper 2001-3071, June 2001.

${ }^{19}$ Sun, Q., Boyd, I. D., and Candler, G. V., "A Hybrid Continuum/Particle Approach for Micro-Scale Gas Flows," Proceedings of the 23th International Symposium on Rarefied Gas Dynamics, Wistler, Canada, 2002.

${ }^{20}$ MacCormack, R. W. and Candler, G. V., "The Solution of the Navier-Stokes Equations Using Gauss-Seidel Line Relaxation," Computers and Fluids, Vol. 17, No. 1, 1989, pp. 135-150.

${ }^{21}$ Gökçen, T. and MacCormack, R. W., "Nonequilibrium Effects for Hypersonic Transitional Flows Using Continuum Approach," AIAA Paper 1989-0461, Jan. 1989.

${ }^{22}$ Sun, Q. and Boyd, I. D., "A Direct Simulation Method for Subsonic, Micro-Scale Gas Flows," Journal of Computational Physics, Vol. 179, No. 2, 2002, to appear.

${ }^{23}$ Dietrich, S. and Boyd, I. D., "Scalar and Parallel Optimized Implementation of the Direct Simulation Monte Carlo Method," Journal of Computational Physics, Vol. 126, 1996, pp. 328-342.

${ }^{24}$ Rovedo, R., Goldstein, D. B., and Varghese, P. L., "Hybrid Euler/Particle Approach for Continuum/Rarefied Flows," Journal of Spacecraft and Rockets, Vol. 35, No. 3, 1998, pp. 258-265.

${ }^{25}$ Garcia, A. L. and Alder, B. J., "Generation of the Chapman-Enskog Distribution," Journal of Computational Physics, Vol. 140, No. 1, 1998, pp. 66-70.

${ }^{26}$ Holden, M. S., "Experimental Database from CUBRC Studies in Hypersonic Laminar and Turbulent Interacting Flows including Flowfield Chemistry," RTO Code Validation of DSMC 
and Navier-Stokes Code Validation Studies CUBRC Report, June 2000.

10 OF 10 